Proceedings of the 2003 IEEE

International Conference on Robotics,Intelligent Systems and Signal Processing

Changsha, China - October 2003

\title{
Active Filters using Single Current Conveyor
}

\author{
Yichuang Sun \\ Department of Electronic, Communication and Electrical Engineering, University of Hertfordshire \\ Hatfield AL109AB,United Kingdom,Email: y.sun@herts.ac.uk
}

Yigang $\mathrm{He}$

Faculty of Electrical and Information Engineering, Hunan University, Changsha 410082, P.R. China E-mail:

\author{
vghe@hnu.net.cn
}

\begin{abstract}
A current to voltage lowpass filter using a single CCII is proposed. This filter can absorb all finite CCII resistances and capacitances and offer both non-inverting and inverting output. Convenient compensation methods exist for gain inaccuracy and excess phase effects. The sensitivities are also very low. The filter is particularly useful for data converting and sampled-data filtering systems as it can fulfil both filtering for antialiasing or smoothing purpose and interfacing between current-mode and voltage-mode systems. We also present a currentmode filter topology using a single dual output CCI, which can realise various types of lowpass, bandpass and highpass filters. Since the output current is taken from one CCII output terminal, the structure is suitable for direct cascade for high-order filter design and less sensitive to the CCII parasitic effects compared with those taking current output from capacitor or resistor branches. Both voltage- and current-mode high-order filters can be readily realized using the proposed two filters as basic sections.
\end{abstract}

\section{Introduction}

Two integrator loop filters using multiple amplifiers have been most widely used [1-4]. Recently active filters using a single amplifier have also received much interest in video signal processing and wireless communication systems $[1,5-7]$. Also, between the two types of topology, a new filter structure which contains two integrator loops, but uses a single amplifier has been proposed [8]. Single amplifier biquads have some attractive features such as low power consumption, low noise, small size, and wide linearity. Active filters using a single op-amp have been used for several decades and most recently they have been used in wireless transceivers, utilising a fully differential voltage buffer [5] or a differential difference amplifier [6]. Active filters based on a single OTA with a single output or multiple outputs, in both the voltage- and current-mode have been systematically investigated in [1]. Single CCII filter design has also received some attention $[7,9,10]$

In switched-current signal processing, antialiasing and post smoothing lowpass filters are needed and also current-mode circuits need to interface with voltagemode circuits. It would be therefore desirable to have a single circuit which can perform both filtering and interfacing (rather than two separate circuits) to reduce the system complexity, power, size and cost. For this purpose, a voltage to current lowpass filter suitable for use at the input of a switched-current circuit has been developed using a single dual output CCII [11-16]. In this paper we propose a current to voltage lowpass filter for use at the output of a switched-current circuit for smoothing filtering and interfacing with the voltagemode system. Not limited to switched-current circuits, the argument also applies to current-mode data converters which also need antialiasing and post smoothing filters and to interface voltage-mode systems. Furthermore, for voltage-mode switched-capacitor circuits and voltage-mode data converters, the proposed filter can also find use at the input of the system for antialiasing lowpass filtering and interfacing with the current-mode system.

Most CCII-RC filters in the literature use single output CCIIs and most current-mode CCII-RC filters take output currents from capacitor or resistor branches. Such current output methods increase the effects of nonideal $\mathrm{CCII}$ input and output impedances 


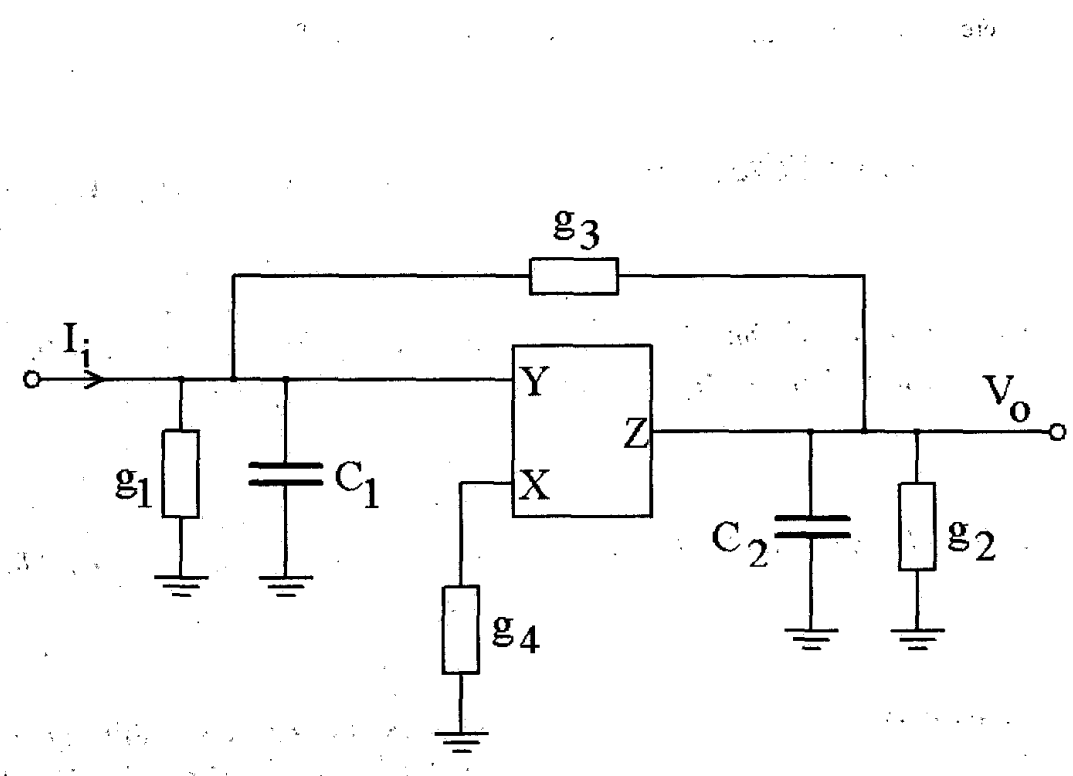

Figure 1. Current to voltage lowpass filter using single $\mathrm{CCII}$

and parasitic node capacitances, and cause coupling if the driven stage does not have zero input impedance in the cascade design. Utilizing the current following and virtual grounding property of the $\mathrm{CCII}$

with $\mathrm{Y}$ terminal grounded, we may insert extra CCIIs into the associated branches to take current outputs. But the nonidealities of these extra CCIIs will severely degrade the filter performance and this method will also increase component count. Multiple output CCIIs can be used to solve the above problems in the generation of current-mode filter structures, since using the multiple output terminals, current output and current feedback can be readily accomplished. A comprehensive set of current-mode two integrator loop filters using dual output CCIIs (DO-CCII) have been proposed [4]. This paper is concerned with currentmode filters using a single DO-CCII.

\section{Current To Voltage Lowpass Filter Using Single CCII}

The proposed current to voltage single CCII lowpass filter is shown in Figure 1. Its transfer function can be derived as

$\frac{V_{0}}{I_{i}}=\frac{g_{3}-g_{4}}{a s^{2}+b s+c}$

There into,

$a=C_{1} C_{2}$

$b=C_{1}\left(g_{2}+g_{3}\right)+C_{2}\left(g_{1}+g_{3}\right)$

$c=\left(g_{1} g_{2}+g_{1} g_{3}+g_{2} g_{3}+g_{3} g_{4}\right)$
The gain polarity can be controlled by $g_{3}$ and $g_{4}$. The non-inverting function can be achieved by selecting $\mathrm{g}_{3}>\mathrm{g}_{4}$, and the inverting function by $\mathrm{g}_{3}<\mathrm{g}_{4}$.

There are choices for the filter design. For example, we may choose $\mathrm{g}_{1}=\mathrm{g}_{2}=\mathrm{g}$ and $\mathrm{C}_{1}=\mathrm{C}_{2}=\mathrm{C}$, then determine $\mathrm{g}$, $\mathrm{g}_{3}$ and $\mathrm{g}_{4}$ for a set $\mathrm{C}$ and the required gain, $\mathrm{K}$, cut-off frequency, $\omega_{0}$ and quality factor, $Q$. The formulae for a normalised frequency, $\omega_{0}=1$, are given by $\mathrm{g}_{3}=\left(1 / 4 \mathrm{Q}^{2}\right.$ 1) $/ K, g_{4}=g_{3}-K^{2} C^{2}$, and $g=C / 2 Q-g_{3}$.

The $\omega_{0}$ and $Q$ sensitivities of the filter are very low. For the above design, for example, the sensitivities of $\omega_{0}$ and $Q$ with respect to $C_{1}$ and $C_{2}$ are -0.5 and 0 , respectively. The sensitivities of $Q$ to $g_{1}, g_{2}$ and $g_{3}$ are equal to 0.5 . All the other sensitivities of $\omega_{0}$ and $Q$ are no larger than 0.5 .

The finite admittances at terminals $Y$ and $Z$ of the CCII can be modelled as $\mathrm{Yy}=\mathrm{Gy}+\mathrm{sCy}$ and $\mathrm{Yz}=\mathrm{Gz}+\mathrm{sCz}$ connected from respective terminals to ground. The finite impedance at terminal $\mathrm{X}$ may be modelled as a series resistance Rx. These finite admittances/impedances will influence filter performance adversely [12]. From the filter structure in Figure 1, we can observe that $\mathrm{Rx}$ can be absorbed by the external resistance connected from terminal $\mathrm{X}$ to ground. Also the external resistors and capacitors at terminals $\mathrm{Y}$ and $\mathrm{Z}$ all are grounded and thus can be used to absorb the CCII finite conductances and capacitances at these terminals. The filter therefore is free from the effects of finite CCII impedances. 


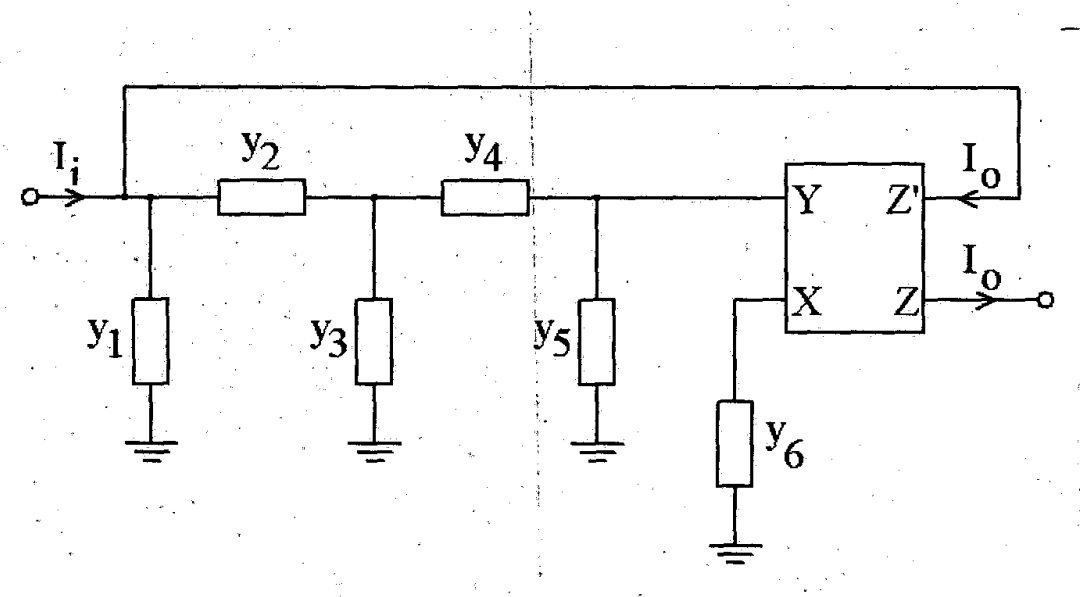

Figure 2 Current-mode filter using single DO-CCII

The CCII is essentially composed of a voltage buffer denoted by $\delta \mathrm{v}$ and $\delta \mathrm{i}$ respectively may be defined as $\mathrm{Vx}=(1+\delta \mathrm{v}) \mathrm{Vy}$, and $\mathrm{Iz}=(1+\delta \mathrm{i}) \mathrm{Ix}[13]$. For the nonideal CCII we can readily prove $I z=\mathrm{g}_{4}(1+\delta \mathrm{i})(1+\delta \mathrm{v}) \mathrm{Vy}=\mathrm{g}_{4}{ }^{\prime} \mathrm{Vy}$, where $\mathrm{g}_{4}{ }^{\prime}=\mathrm{g}_{4}(1+\delta \mathrm{i})(1+\delta \mathrm{v})$. Hence the effect of the tracking errors may be equivalently considered to cause the change in the conductance of the $\mathrm{g}_{4}$ resistor. Therefore the effects of CCII buffering errors on filter performances can be very easily evaluated by directly substituting: $\mathrm{g}_{4}$ ' for $\mathrm{g}_{4}$ in the transfer function.

To eliminate the effects of buffering errors we can add a resistor in parallel or series with the $\mathrm{g}_{4}$ resistor to accommodate the buffering errors [13]. When $(1+\delta \mathrm{v})(1+\delta \mathrm{i})<1$, parallel compensation should be adopted. The corresponding compensation equation is then readily derived as $(1+\delta \mathrm{v})(1+\delta \mathrm{i})\left(\mathrm{g}_{4}+\mathrm{g}_{4 \mathrm{cp}}\right)=\mathrm{g}_{4}$, from which we can determine the parallel compensation conductance as $\mathrm{g}_{4 \mathrm{cp}}=\mathrm{g}_{4}[1 /(1+\delta \mathrm{v})(1+\delta \mathrm{i})-1]$. When $(1+\delta v)(1+\delta i)>1$, series compensation should be utilized and in this case the compensation equation becomes $(1+\delta \mathrm{v})(1+\delta \mathrm{i}) \mathrm{g}_{4} \mathrm{~g}_{4 \mathrm{cs}} /\left(\mathrm{g}_{4}+\mathrm{g}_{4 \mathrm{cs}}\right)=\mathrm{g}_{4}$, which gives the series compensation conductance as

$\mathrm{g}_{4 \mathrm{cs}}=\mathrm{g}_{4} /[(1+\delta \mathrm{v})(1+\delta \mathrm{i})-1]$.

The excess phase of the CCII will pose a stability problem at higher frequencies [12]. For the given CCII, to extend the working frequency of the filter, we must overcome the finite bandwidth effects. The passive compensation technique using a capacitor can be used. For the CCII, suppose that $\mathrm{Vx} / \mathrm{Vy}=1 /\left(1+\mathrm{s} \tau_{\mathrm{v}}\right)$, and $\mathrm{Iz} / \mathrm{Ix}=$ and a current buffer. Voltage and current tracking errors $1 /\left(1+s \tau_{i}\right)$, then Iz=YVy, where $\mathrm{Y}=\mathrm{g}_{4} /\left[\left(1+s \tau_{\mathrm{v}}\right)\left(1+\mathrm{s} \tau_{\mathrm{i}}\right)\right] \cong$ $\mathrm{g}_{4} /\left[1+\mathrm{s}\left(\tau_{\mathrm{v}}+\tau_{\mathrm{i}}\right)\right]$ for small $\tau_{\mathrm{v}}$ and $\tau_{\mathrm{i}}$.

The effect is equivalent to a series of a resistor with an inductor with inductance equal to $\mathrm{L}=\left(\tau_{\mathrm{v}}+\tau_{\mathrm{i}}\right) / \mathrm{g}_{4}$. To eliminate the excess phase effect, one can use a capacitor in parallel with the $\mathrm{g}_{4}$ resistor. With the compensation capacitor $\mathrm{C}_{\mathrm{c}}$, we can write $\mathrm{Iz}=\left(\mathrm{g}_{4}+\mathrm{s} \mathrm{C}_{\mathrm{c}}\right) /\left[1+\mathrm{s}\left(\tau_{\mathrm{v}}+\tau_{\mathrm{i}}\right)\right] \mathrm{Vy}$, which leads to $\mathrm{C}_{\mathrm{c}}=$ $\mathrm{g}_{4}\left(\tau_{\mathrm{v}}+\tau_{\mathrm{i}}\right)$.

\section{Current-Mode Active Filters Using Single Dual-Output CCII}

The current-mode filter configuration using a single DOCCII is shown in Figure 2. Its general current transfer function can be derived as

$$
\begin{aligned}
& \frac{I_{0}}{I_{I}}=\frac{y_{2} y_{4} y_{6}}{y_{1} y_{4} d+y_{2} y_{4} e+y_{1} y_{5} f+y_{2} y_{3} y_{5}} \\
& d=y_{2}+y_{3}+y_{5} \\
& e=y_{3}+y_{5}+y_{6} \\
& f=y_{2}+y_{3}
\end{aligned}
$$

Many types of filter can be realised by this circuit. Some are given in Table 1.

In the literature current-mode CCII-RC filters often take output currents from capacitor or resistor branches. Such current output methods are not suitable for 


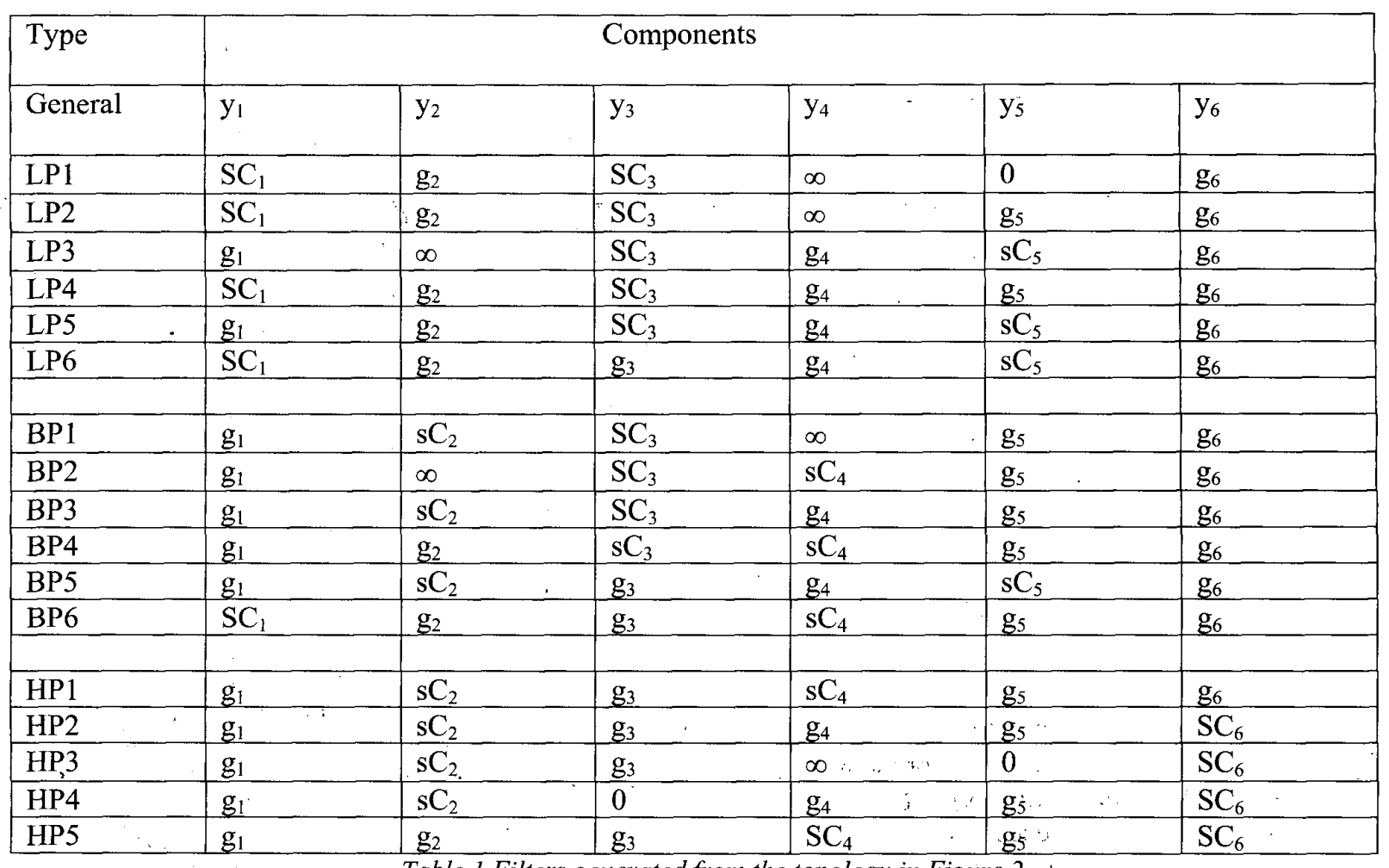

\footnotetext{
$\therefore \quad$ Table 1 Fille
}

connection as coupling will occur if the driven stage does nothave zerö input impedance. Utilizing the current following and virtual grounding property of the CCII with Y terminal grounded, we may insert extra CCIIs into the associated branches to take current outputs. But the nonidealities of these extra CCIIs will severely. degrade the filter performance and this method will also increase component count. The proposed filter structure in Figure 2 uses a multiple current output CCII and the filter current output can be taken directly from the output terminal.

For high-order filter design using the current transfer function, the proposed current to current biquad in Figure 2 can be cascaded directly. For the realization of a high-order lowpass voltage transfer function, we may start with a voltage to current section such as the one in [11], cascade it with the current to current sections in Figure 2 and end up with the current to voltage section in Figure 1. Note that in some applications, to take the advantage of high performance of current-mode filters, people do design high-order voltage filters in this.way, rather than by cascading voltage to-voltage sections.

\section{Conclusions}

We have presented a new current to voltage lowpass filter using a single CCII. The filter has many attractive features such as low sensitivity', parasitic free, capable of both inverting and non-inverting output, easy to compensate for CCII gain inaccuracies and excess phase. It is particularly useful for sampled-data filtering and data converting systems as it can simultaneously perform lowpass filtering and interfacing between the current- and voltage-mode systems. A current-mode filter architecture using a single dual output $\mathrm{CCII}$ has also been proposed. The filter can be 
cascaded directly due to the output taken from the CCII output terminal and has lower parasitic effects and' lower component count than those taking current output' from capacitor or resistor branches. We have shown the range of filter functions which are supported by the configuration. Using the two filters proposed as basic sections, both voltage and current high order transfer functions can be realised by cascading them. Finally, it should be noted that in practical implementations of these single amplifier filters, resistors are often replaced by MOSFETs in triode region and balanced/fully: differential structure are used as is well known in MOSFET-C filter design [1].

\section{Acknowledgement}

The work is supported by the Natural Science Funding Council of China under Grant No. 50277010 , Doctoral Discipline Special Fund of Colleges and Universities of China (20020532016) and the Fund of Outstanding Young Scientist of Hunan University of China.

\section{References}

[1] T. Deliyannis, Y. Sun and J. K. Fidler, Continuous-time Active Filter Design, CRC Press, Florida, USA, January 1999, ISBN: 0-8493-7893-

[2] C. Toumazou and F. J. Lidgey, "Universal active filter using current conveyors," Electron. Lett., vol.22, pp.662-664, 1986.

[3] Y. Sun and J. K. Fidler, "Versatile active biquad based on second-generation current conveyors," Int. J. Electron., vol.76, pp.91-98, 1994. ro

[4] Y. Sun and B. Jefferies, "Current-mode biquadratic. filters using dual output current conveyeors," Proc. IEEE'Int. Conf. on Electronics, Circuits and Systems, pp.135-138, Portugal, Sept. 1998.
[5] H. Elwan, M. I. Younus and M. Ismail, "A buffer based CMOS baseband chain for bluetooth receiver," IEEE ISCAS 2002, Vol.II, pp.13-16, Arizona, USA, 2002.

[6] H. Elwan. H. Alzaher and M. Ismail, "A new generation of global wireless compatibility," IEEE Circuits and Devices Magazine, January, pp.2-19, 2001.

[7] H. P. Schmid and G. S. Moschytz, "Active MOSFET-C single-amplifier biquadratic filters for video frequencies". in Special Issue on High-frequency Integrated Analogue Filters, IEE Proc. Circuits, Devices and Systems, 'Vol. 147, No. 1, pp. 35-41, Feb. 2000.

[8] Y. Sun and C. Hill, "Low power fully differential CMOS filter for video frequencies," IEEE Transactions on Circuits and Systems, Part-II: Analog and Digital Signal Processing, vol.48, No.12, 2001.

[9] S. I. Liu and H. W. Tsao, " The single CCII biquads with high-input impedances," IEEE Trans CAS Vol. 38, No.4, pp. 456-461, 1991 .

[10] C. P. Chong and K. C. Smith, "Biquadratic filter sections employing a single current conveyor," Electronics Letters, vol. 22, No.22, pp. $11621164,1986$.

[11] R. A. H. Balmford, W. Redman-White and J. B. Hughes, "Low distortion interfaces and antialias filters for switched current applications," Proc.IEEE Int. Symp. Circuits Systems, vol.5, pp.285-288, 1994.

[12] A. Fabre, O. Saaid and H. Barthelemy, "On the frequency limitations of the circuits based on second generation current conveyors," Analog Integrated Circuits and Signal Processing, vol. 7, pp.113-129, 1995.

[13] Y. Sun and J. K. Filder, "Analysis of current conveyor error effects in signal processing circuits," Int. J. Cir. Theor. Appl., vol.24, pp.479-487, 1996.

[14]Y.He,J.Jiang, J.Wu, Universal active current-mode filter, ACTA ELECTRONICA SINICA,Vol.27,No.11,pp21-23,1999

[15]Y.He,Current mode continuous-time filters based on MOCC,Journal of China institute of communications, Vol.21,No.3,pp88-91,2000

[16] Y.He,J.Jiang,J.Wu,Fully differential $4^{\text {th }}$-order bessel filter accurately designed with group delay, ACTA ELECTRONICA SINICA,Vol.30,No.2,pp249-251,1999 